\title{
Fetal cells in maternal blood for prenatal diagnosis: a love story rekindled
}

\author{
Ripudaman Singh ${ }^{*}{ }^{1}$, Lotte Hatt ${ }^{1}$, Katarina Ravn ${ }^{1}$, Ida Vogel ${ }^{2}$, Olav Bjørn Petersen ${ }^{3}$, Niels \\ Uldbjerg $^{3}$ \& Palle Schelde ${ }^{1}$ \\ ${ }^{1}$ ARCEDI Biotech ApS, Dandyvej 19, 7100, Vejle, Denmark \\ 2Department of Clinical Genetics. Aarhus University Hospital, Aarhus, Palle-Juul Jensens Boulevard 99, 8200, Aarhus N, Denmark \\ ${ }^{3}$ Department of Obstetrics \& Gynecology, Aarhus University Hospital, Aarhus, Palle-Juul Jensens Boulevard 99, 8200, Aarhus N, \\ Denmark \\ * Author for correspondence: Tel.: +45 28717 247; rs@arcedi.com
}

First draft submitted: 15 February 2017; Accepted for publication: 31 May 2017; Published online: 15 June 2017

Keywords: cell-based noninvasive prenatal diagnosis • fetal cells in maternal blood • noninvasive prenatal testing

\section{Prolog}

For decades it has been common knowledge that some cells from the developing fetus make their way into maternal circulation, and that this process starts in the early weeks of first trimester. Naturally, when alternative technologies for prenatal diagnosis were being explored, the use of fetal cells for noninvasive prenatal diagnosis came on top of the list. After years of intermediate success and failure, the interest in fetal cells circulating in maternal blood has come full circle. Recent advances in single fetal cell genetic analyses have invigorated interest in fetal cells for prenatal diagnostics. However, for this interest to sustain, we propose a list of best practices that have their origins in the lessons learned from past successes and failures.

\section{Fetal cells in maternal blood: a brief history}

Fetal nucleated red blood cells (nRBCs) were first reported in maternal circulation in a paper published in The Lancet in 1959 [1]. However, it was not before 1969 when the potential of circulating fetal cells in noninvasive prenatal diagnosis was proposed [2]. A decade later, the first report describing a method to successfully enrich fetal lymphocytes from maternal circulation was published [3]. Following these initial studies the so-called 'golden era' of circulating fetal cell research spanned for 10 years from 1993 to 2003 (Figure 1). One of the largest multicenter initiatives to enrich and use fetal cells for aneuploidy detection was taken on by the National Institute of Child Health and Human Development Fetal Cell Isolation Study, the NIFTY trial. Ten different research centers used different protocols and methods to isolate nRBCs from maternal circulation. However, this initiative did not bear a fruit of cell-based noninvasive prenatal diagnosis (cbNIPD) because of its inability to isolate these cells from every pregnancy and also due to a high false-positive rate of gender and aneuploidy detection [4]. This eventually led to dampening of the enthusiasm surrounding circulating fetal cells.

Lately, the fetal cbNIPD has again been in focus, pushed by advances in technologies aiming at single-cell genetic analyses. In this second coming of the fetal cells, it is pertinent that some of the concerns that were raised earlier are addressed. This will rectify and counterbalance the skepticism that has built around using fetal cells for prenatal diagnostics. Else the risk that the interest in circulating fetal cells will again be pushed into oblivion is high.

Knowledge of the fetal cell type and the markers that these rare cells express have been the holy grail of realizing a cbNIPD. Moreover, the attempts to successfully isolate fetal cells have been made difficult by the rarity of these cells in the maternal circulation. Some calculations put these numbers to as low as one cell in $1 \mathrm{ml}$ of maternal whole blood [5].

\section{Checklist for cbNIPD}

If a technology claims to perform cbNIPD, there are certain criteria that it should fulfill. We propose the following checklist that needs to be fulfilled to maximize the chances of a successful cbNIPD. 


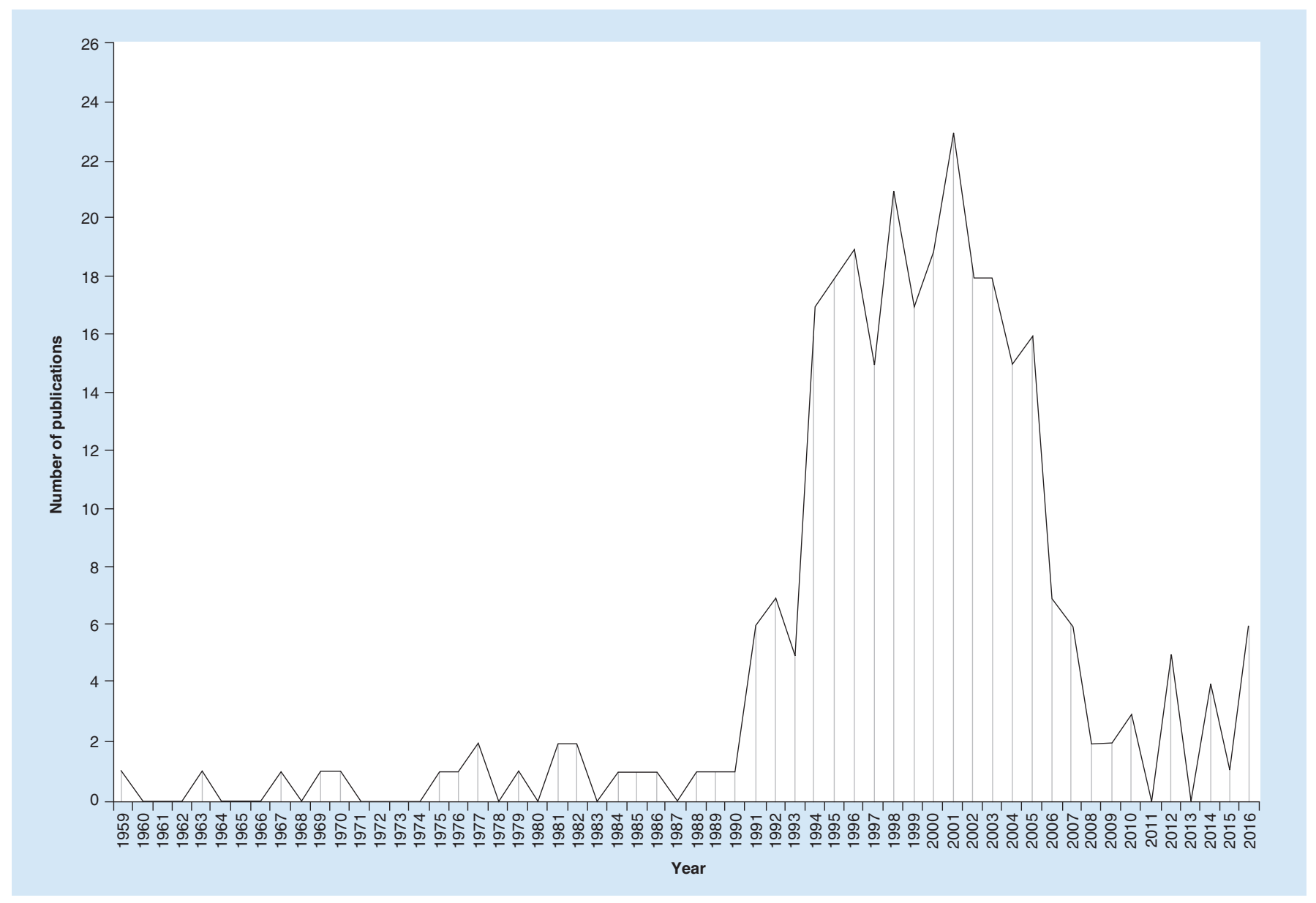

Figure 1. The number of publications targeting fetal cells in maternal blood. Graph generated from citations in Pubmed.

- The technology should target specific cell type(s).

- There should be antibodies that are both sensitive and specific for those cell types.

- Technology should be platform and parameter independent.

- After identifying the true fetal cells it should be possible to get access to the cells for downstream applications.

- The fidelity of the DNA from enriched fetal cells should be high so that genetic analyses using chromosomal microarray (CMA) or next-generation sequencing (NGS) can be performed.

\section{Fetal cell type \& markers}

The identity of fetal cells that circulate in pregnant women's blood and the biomarkers that they express have been the holy grail of fetal cell research. Herzenberg et al. in 1979 were the first to demonstrate the enrichment of fetal leucocytes from maternal blood by using immunomagnetic cell sorting technique [3]. Since then other cell types, namely $\mathrm{nRBCs}$ and trophoblasts have been identified in maternal circulation.

\section{Nucleated red blood cells}

Historically, nRBCs have been the most commonly targeted fetal cell type for NIPD, but with only limited success mainly due to low specificity markers and also due to low cell numbers in maternal blood. In 1998, a study reported the first noninvasive prenatal detection of trisomy 13 (T13) based on nRBCs obtained prior to Chorionic Villi Sampling (CVS) in the first trimester and identified by fetal hemoglobin (HbF) staining [6]. However, HbF staining did not give a $100 \%$ specificity toward fetal cell recognition, nor when an anti-CD45 antibody was applied for elimination of leukocytes [7]. Parano et al. were also able to identify common aneuploidies such as trisomy 21 (T21), T13 and XXY on nRBCs from the second trimester pregnancies, and reported a minimized maternal contamination when using a micromanipulator to isolate the fetal cells [8]. More specific enrichment procedures of nRBCs were 
pursued by targeting the iron-binding protein transferrin $(\mathrm{CD} 71)[9,10]$ or targeting both $\mathrm{CD} 71$ and glycophorin $\mathrm{A}$ (Gly A) using a magnetic-activated cell sorting platform. Following CD71 or Gly A enrichment and HbF staining, Martel-Petit et al. identified nRBCs, which revealed a fetus affected with cystic fibrosis. Nonetheless, problems concerning the specificity (false-positive staining) persisted [11]. The large NIFTY trial utilized antibodies targeting either $\mathrm{HbF}, \mathrm{HbF}$ and $\mathrm{CD} 45$, or $\mathrm{CD} 71$, but the study resulted in poor detection rates of aneuploidies and the correct fetal gender [4]. In 2009, a comparison between different enrichment antibodies was pursued by Souza et al. who found that a combination of antibodies targeting CD45, $\mathrm{HbF}$ and CD71 yielded the highest specificity of fetal nRBCs but also a lower number of fetal cells, still leaving the use of nRBCs in cbNIPD with limited success [12]. Christensen and colleagues found that embryonic hemoglobin is more specific toward fetal nRBCs than HbF [13], but the group also concluded that only a small fraction of circulating fetal cells are of erythroblastic origin and therefore not the optimal cell type for cbNIPD $[13,14]$.

\section{Trophoblasts}

In recent years, trophoblasts have gained more attention and proven to be relevant targets in cbNIPD. Cacheux et al. were the first to report a chromosomal aberration (XYY) identified from trophoblasts retrieved from the maternal circulation using immunomagnetic depletion of leukocytes, and flow cytometry with the antitrophoblasts antibodies GB17 and GB25. However, only 4.25\% of the nuclei analyzed by FISH showed a Y-signal [15]. Human leukocyte antigen $G$ has also been successfully used to enrich circulating trophoblasts from maternal circulation and use those cells for aneuploidy detection [16-18]. Other studies have relied on size-based isolation of fetal trophoblastic cells and used those cells for prenatal diagnosis [19]. Our group established fetal gene expression profile to generate knowledge about the type of fetal cells that circulate in maternal blood and the biomarkers that they express. In the first, nontargeted approach, fetal cells were isolated either by using no-enrichment method, or by depletion of maternal leucocytes on a magnetic-activated cell sorting platform. Twenty-three fetal cells collected this way were then run on PIQOR ${ }^{\mathrm{TM}}$ stem cell microarray, which demonstrated an overexpression of endothelial and placental genes in fetal cells compared with the maternal cells [20].

Based on the results from the first array, we did a second, more targeted gene expression array analysis on 198 circulating fetal cells that were enriched using endoglin. This analysis revealed that over half of the overexpressed genes in fetal cells were specific in extravillous trophoblasts (EVTs), indicating that this cell type could be a target in cbNIPD. In the placenta, some cytotrophoblasts from the column of the anchoring villi invade the maternal decidua [21]. Eventually we know that these invading trophoblasts line the spiral arteries, glands and veins and could be the source of fetal cells in maternal circulation [22]. This method of targeting EVTs has been very reliable in enriching fetal cells from every pregnant sample. Fetal cells enriched this way have subsequently been used to detect chromosomal and subchromosomal changes in the fetal genome, thus confirming EVTs to be relevant targets in $\operatorname{cbNIPD}[17]$.

\section{Platform \& parameter independent cbNIPD technology}

In order for a cell-based technology to be used as a routine prenatal test some of the parameters that are worth considering are related to blood sample collection, handling and processing, and to check whether any of these affect the retrieval of fetal cells. Type of the blood collection tube, time before blood processing and transportation of the samples may be some issues that can affect the enrichment of rare fetal cells and their usability for prenatal diagnosis. Dukes et al. tested the hypothesis that the time elapsed between blood sampling and enrichment of fetal cells affects the recovery of fetal cells from maternal blood [23]. They found that there was an increase in the number of nRBCs in the samples processed after 18-24 $\mathrm{h}$ as compared with $4 \mathrm{~h}$ after sampling. Using the method to enrich fetal cells recently described by us [17], we have performed several experiments testing the stability of blood samples and found that after drawing the blood it can be kept in the lab or transported by air for up to 48 $\mathrm{h}$ before processing, without affecting the fetal cell recovery [UNPUBLISHED DATA]. On one hand these data reflect the robustness of the technologies and on the other hand show the stability of fetal cells in maternal circulation.

\section{Fetal cell accessibility}

The era of only counting immobilized fetal cells on glass slides without using them for molecular genetic analyses is over. Moreover, the new molecular genetic techniques require that the fetal cells are captured in a PCR tube so that downstream genetic analysis can be performed on them. Single cell manipulation has also been pushed by the research on circulating tumor cells, and there are various technologies that can be used to get access to single cells. 
We have tried various capillary-based instruments (CellCelector from Automated Lab Solutions GmbH, Germany, and CyteFinder \& CytePicker from Rarecyte, WA, USA) and laser capture microdissection method (Carl Zeiss AG, Germany) to get access to fetal cells. Both the technologies have their merits and demerits. Laser capture microdissection method is quicker but less delicate on cells. However, the capillary-based cell collection is more delicate on the cells but is time consuming.

\section{DNA fidelity}

In order to apply rare fetal cells for downstream genetic analyses such as CMA or NGS, the scarce DNA genome equivalents needs to be amplified to more copies. It is crucial that this whole-genome amplification (WGA) is of high fidelity to incorporate a minimum of representational bias. Another important concern is the risk of DNA contamination to the few picograms of fetal DNA during the WGA process from both the personnel and the surroundings. Therefore, WGA should be managed in a dedicated clean room with utmost care.

At present there is a range of commercially available WGA kits catering rare cells, using different chemistries and methodological strategies. Broadly the WGA can be divided into three categories: PCR-based WGA (mainly degenerate-oligonucleotide-primed PCR), multiple displacement amplification WGA, which is isothermal amplification and multiple annealing and looping-based amplification cycles WGA [24,25]. The different chemistries possibly will yield different performances suitable for different applications. For prenatal diagnosis, detection of the common genomic aberrations such as aneuploidies, as well as copy number variations (CNVs; microdeletions and microduplications) are important. The PCR-based WGA have shown the most promising detection of CNVs.

In concordance with this, Normand et al. tested three commercially available, PCR-based WGA kits: Ampli1 (Silicon Biosystems, Bologna, Italy), Picoplex (Rubicon, Ann Arbor, MI, USA) and GenomePlex (Sigma Aldrich, Darmstadt, Germany) for molecular genotyping and CMA analysis of single cells, using lymphoblastoid cell lines harboring various known pathogenic CNVs. They were able to demonstrate sufficient WGA quality to detect $>1$ MB copy number changes using a catalog $180 \mathrm{~K}$ Agilent array for all three WGA kits. Importantly, fixation and permeabilization, reagents in fetal cell enrichment and staining, were also tested and found to be compatible with all three WGA Kits. Ampli 1 WGA was however found to be the most sensitive kit being able to detect a $1.3 \mathrm{MB}$ (CMT1A) duplication and a 0.6 MB (MECP2) duplication consistently in three individual fixed cells.

Within the last year, it has been possible to enrich fetal cells from maternal blood, pick them individually and obtain a sufficient quality of the whole genome amplified DNA to provide the correct prenatal diagnosis corresponding to the CMA results obtained from either CVS of amniocentesis.

In a recent study published in Prenatal Diagnosis we, among others, have successfully demonstrated an extra copy of chromosome 21 in a female fetus, as well as the expected copy number differences for the $\mathrm{X}$ and $\mathrm{Y}$ chromosomes compared with gender-mismatched reference DNA. In another pregnancy carrying a fetus which was mosaic for monosomy $\mathrm{X}$ and a cell line with telomeric deletions on chromosome $\mathrm{X}$, we could detect a complete loss of chromosome $\mathrm{X}$ and subtelomeric deletion in the DNA from two individual fetal cells respectively [26]. The results were confirmed by CMA and NGS on an amniocentesis sample.

In a study by Breman $e$ t al. they demonstrated a range of aneuploidies, including T21, T13, T18 and a 47 XXY, on single fetal cells enriched from maternal blood. They could also detect a subchromosomal aberration, a $2.7 \mathrm{MB}$ de novo deletion in two individual fetal cells [27].

Both of these latest studies demonstrating aneuploidies and CNVs on single or few fetal trophoblastic cells, illustrate that it is feasible to amplify DNA from circulating fetal cells in a quality sufficient for CMA or NGS analysis for prenatal diagnosis. Further optimizations of both the WGA and the analysis platforms, CMA and NGS, should permit an even higher resolution of fetal anomaly detection.

\section{Positioning of a cell-based diagnostic technology}

Technologically, if all the dots leading to a successful enrichment and analysis of fetal cells are connected, how would such a technology fit into the current prenatal diagnostics arena? Currently the NIPT field has largely been dominated by methods targeting cell-free fetal DNA in the maternal blood. Even though these methods can reliably detect incidence of common aneuploidies (T21, T13 and T18), they have been ineffective in detecting CNVs, which constitute another major chunk of prenatal abnormalities [28]. This is mainly due to the fact that fetal DNA circulating in maternal blood is fragmented and is mixed with maternal DNA. Intact fetal cells circulating in maternal blood can mitigate this shortcoming of the cell-free NIPT (cfNIPT), because they are the sources of pure fetal genomes. 
The current pregnancy risk stratification is based on the risk of carrying a T21 fetus. Hence, women labeled as 'low risk' have a low risk of carrying a fetus affected by Down's syndrome, but typically have a higher risk for deletion/duplication syndromes. A current estimate puts the residual risk of deletion/duplication syndromes to be 1:270 [29]. Thus, cfNIPT is a wrong test to be applied on 'low-risk' women. cfNIPT for 'high-risk' women is more productive, but it can induce a diagnostic delay, since positive events will have to be confirmed by either CVS or amniocentesis. On the other hand fetal cell-based approach may prevent this delay. Due to these advantages, fetal cells have the potential to be used in a diagnostic test. In order to achieve this, clinical trials need to be run which will determine sensitivity, specificity and positive predictive value of a cell-based approach. A clinical validation of a cell-based technology will also address important issues surrounding other biological phenomena such as vanishing twin pregnancies and mosaicism. Moving ahead, the cost of a cell-based diagnostic test is another issue that would need to be addressed. The field of cfNIPT has been crowded by many companies now offering a screening test for common aneuploidies. This has also pulled down the cost of the test. However, scaling and automation of the cbNIPD are few factors that will have to be considered for the cost of such a test to match with that of cfNIPT. This is something that is already being actively pursued.

\section{Epilogue}

Fetal cells do circulate in pregnant women's blood. Even though rare in numbers, these cells can be enriched from maternal circulation and used for knowing the genetic status of the fetus. This opens up immense opportunities for prenatal screening. Invasive methods such as CVS and amniocentesis still remain the strongest and most reliable methods of prenatal diagnosis of chromosomal aberrations as well as CNVs. However, these invasive procedures not only pose a small risk of spontaneous abortions, but also instill fear and discomfort among the pregnant couple.

In the last couple of years data from some studies have shown that a cbNIPD is clinically viable. Scientific evidence has revived a growing belief that not only is isolation and identification of circulating fetal cells viable but also that subsequent genetic analysis is possible. Based on these findings, in the near future cbNIPD could be placed in the fetal diagnostic arena as a superior alternative to cfNIPT, hence downscaling the need for invasive testing. Looking ahead, in the long run, cbNIPD has the potential to make invasive sampling for prenatal diagnosis obsolete.

\section{Financial \& competing interests disclosure}

Ripudaman Singh, Lotte Hatt, Katarina Ravn and Palle Schelde are employed by ARCEDI Biotech ApS, a Danish biotech company that has developed technologies for circulating fetal cell isolation, detection and analysis. The authors have no other relevant affiliations or financial involvement with any organization or entity with a financial interest in or financial conflict with the subject matter or materials discussed in the manuscript apart from those disclosed

No writing assistance was utilized in the production of this manuscript.

\section{Open access}

This work is licensed under the Attribution-NonCommercial-NoDerivatives 4.0 Unported License. To view a copy of this license, visit http://creativecommons.org/licenses/by-nc-nd/4.0/

\section{References}

1 Zipursky A, Hull A, White FD, Israels LG. Foetal erythrocytes in the maternal circulation. Lancet 1(7070), 451-452 (1959).

2 Walknowska J, Conte FA, Grumbach MM. Practical and theoretical implications of fetal-maternal lymphocyte transfer. Lancet 1(7606), 1119-1122 (1969).

3 Herzenberg LA, Bianchi DW, Schroder J, Cann HM, Iverson GM. Fetal cells in the blood of pregnant women: detection and enrichment by fluorescence-activated cell sorting. Proc. Natl Acad. Sci. USA 76(3), 1453-1455 (1979).

4 Bianchi DW, Simpson JL, Jackson LG et al. Fetal gender and aneuploidy detection using fetal cells in maternal blood: analysis of NIFTY I data. National Institute of Child Health and Development Fetal Cell Isolation Study. Prenat. Diagn. 22(7), 609-615 (2002).

5 Hamada H, Arinami T, Kubo T, Hamaguchi H, Iwasaki H. Fetal nucleated cells in maternal peripheral blood: frequency and relationship to gestational age. Hum. Genet. 91(5), 427-432 (1993).

6 Oosterwijk JC, Mesker WE, Ouwerkerk-Van Velzen MC et al. Prenatal diagnosis of trisomy 13 on fetal cells obtained from maternal blood after minor enrichment. Prenat. Diagn. 18(10), 1082-1085 (1998).

7 de Graaf IM, Jakobs ME, Leschot NJ et al. Enrichment, identification and analysis of fetal cells from maternal blood: evaluation of a prenatal diagnosis system. Prenat. Diagn. 19(7), 648-652 (1999). 
8 Parano E, Falcidia E, Grillo A et al. Noninvasive prenatal diagnosis of chromosomal aneuploidies by isolation and analysis of fetal cells from maternal blood. Am. J. Med. Genet. 101(3), 262-267 (2001).

9 Durrant LG, Martin WL, McDowall KM, Liu DT. Isolation of fetal trophoblasts and nucleated erythrocytes from the peripheral blood of pregnant women for prenatal diagnosis of fetal aneuploides. Early Hum. Dev. 47(Suppl.), S79-S83 (1996).

10 Reading JP, Huffman JL, Wu JC et al. Nucleated erythrocytes in maternal blood: quantity and quality of fetal cells in enriched populations. Hum. Reprod. 10(9), 2510-2515 (1995).

11 Martel-Petit V, Petit C, Marchand M et al. Use of the Kleihauer test to detect fetal erythroblasts in the maternal circulation. Prenat. Diagn. 21(2), 106-111 (2001).

12 D'Souza E, Ghosh K, Colah R. A comparison of the choice of monoclonal antibodies for recovery of fetal cells from maternal blood using FACS for noninvasive prenatal diagnosis of hemoglobinopathies. Cytometry B Clin. Cytom. 76(3), 175-180 (2009).

13 Christensen B, Philip J, Lykke-Hansen L, Kolvraa S. Sensitivity and specificity of the identification of fetal cells in maternal blood by combined staining with antibodies against beta-, gamma- and epsilon-globin chains. Fetal Diagn. Ther. 18(6), 479-484 (2003).

14 Kolvraa S, Christensen B, Lykke-Hansen L, Philip J. The fetal erythroblast is not the optimal target for non-invasive prenatal diagnosis: preliminary results. J. Histochem. Cytochem. 53(3), 331-336 (2005).

15 Cacheux V, Milesi-Fluet C, Tachdjian G et al. Detection of 47,XYY trophoblast fetal cells in maternal blood by fluorescence in situ hybridization after using immunomagnetic lymphocyte depletion and flow cytometry sorting. Fetal Diagn. Ther. 7(3-4), 190-194 (1992).

16 Guetta E, Gutstein-Abo L, Barkai G. Trophoblasts isolated from the maternal circulation: in vitro expansion and potential application in non-invasive prenatal diagnosis. J. Histochem. Cytochem. 53(3), 337-339 (2005).

17 Kolvraa S, Singh R, Normand EA et al. Genome-wide copy number analysis on DNA from fetal cells isolated from the blood of pregnant women. Prenat. Diagn. 36(12), 1127-1134 (2016).

18 van Wijk IJ, Griffioen S, Tjoa ML et al. HLA-G expression in trophoblast cells circulating in maternal peripheral blood during early pregnancy. Am. J. Obstet. Gynecol. 184(5), 991-997 (2001).

19 Mouawia H, Saker A, Jais JP et al. Circulating trophoblastic cells provide genetic diagnosis in 63 fetuses at risk for cystic fibrosis or spinal muscular atrophy. Reprod. Biomed. Online 25(5), 508-520 (2012).

20 Brinch M, Hatt L, Singh R et al. Identification of circulating fetal cell markers by microarray analysis. Prenat. Diagn. 32(8), 742-751 (2012).

21 Hatt L, Brinch M, Singh R et al. Characterization of fetal cells from the maternal circulation by microarray gene expression analysis-could the extravillous trophoblasts be a target for future cell-based non-invasive prenatal diagnosis? Fetal Diagn. Ther. 35(3), 218-227 (2014).

22 Moser G, Weiss G, Sundl M et al. Extravillous trophoblasts invade more than uterine arteries: evidence for the invasion of uterine veins. Histochem. Cell Biol. 147(3), 353-366 (2016).

23 Dukes KA, Sullivan LM, Lewis D et al. The effect of the elapsed time between blood draw and processing on the recovery of fetal cells from maternal blood. J. Soc. Gynecol. Investig. 11(3), 154-165 (2004).

24 Hou Y, Wu K, Shi X et al. Comparison of variations detection between whole-genome amplification methods used in single-cell resequencing. Gigascience 4, 37 (2015).

25 Huang L, Ma F, Chapman A, Lu S, Xie XS. Single-cell whole-genome amplification and sequencing: methodology and applications. Annu. Rev. Genomics Hum. Genet. 16, 79-102 (2015).

26 Kolvraa S, Singh R, Normand EA et al. Genome-wide copy number analysis on DNA from fetal cells isolated from the blood of pregnant women. Prenat. Diagn. 36(12), 1127-1134 (2016).

27 Breman AM, Chow JC, U'Ren L et al. Evidence for feasibility of fetal trophoblastic cell-based noninvasive prenatal testing. Prenat. Diagn. 36(11), 1009-1019 (2016).

28 Petersen OB, Vogel I, Ekelund C, Hyett J, Tabor A. Potential diagnostic consequences of applying non-invasive prenatal testing: population-based study from a country with existing first-trimester screening. Ultrasound Obstet. Gynecol. 43(3), 265-271 (2014).

29 Srebniak MI, Joosten M, Knapen M et al. Frequency of submicroscopic chromosome aberrations in pregnancies without increased risk for structural chromosome aberrations - a systematic review of literature and meta-analysis. Ultrasound Obstet. Gynecol. doi:10.1002/uog.17533 (2017) (Epub ahead of print). 